\title{
Procedural sedation for direct current cardioversion: a feasibility study between two management strategies in the emergency department
}

\author{
Giulia Stronati ${ }^{1}$, Alessandro Capucci ${ }^{1}$, Antonio Dello Russo ${ }^{1}$, Erica Adrario ${ }^{2}$, Andrea Carsetti ${ }^{2}$, Michela Casella ${ }^{1}$,
} Abele Donati ${ }^{2}$ and Federico Guerra ${ }^{1 *}$ (D)

\begin{abstract}
Background: A cardiologist-only approach to procedural sedation with midazolam in the setting of elective cardioversion (DCC) for AF has already been proven as safe as sedation with propofol and anaesthesiologist assistance. No data exist regarding the safety of such a strategy during emergency procedures. The aim of this study is to compare the feasibility of sedation with midazolam, administered by a cardiologist, to an anaesthesiologist-assisted protocol with propofol in emergency DCC.
\end{abstract}

Methods: Single centre, prospective, open blinded, randomized study including all consecutive patients admitted to the Emergency Department requiring urgent or emergency DCC. Patients were randomized in a 1:1 fashion to either propofol or midazolam treatment arm. Patients in the midazolam group were managed by the cardiologist only, while patients treated with propofol group underwent DCC with anaesthesiologist assistance.

Results: Sixty-nine patients were enrolled and split into two groups. Eighteen patients (26.1\%) experienced periprocedural adverse events (bradycardia, severe hypotension and severe hypoxia), which were similar between the two groups and all successfully managed by the cardiologist. No deaths, stroke or need for invasive ventilation were registered. Patients treated with propofol experienced a greater decrease in systolic and diastolic blood pressure when compared with those treated with midazolam.

As the procedure was shorter when midazolam was used, the median cost of urgent/emergency DCC with midazolam was estimated to be $129.0 €$ (1st-3rd quartiles 114.6-151.6) and $195.6 €(1 \mathrm{st}-3 \mathrm{rd}$ quartiles 147.3-726.7) with propofol $(p<.001)$.

Conclusions: Procedural sedation with midazolam given by the cardiologist alone was feasible, well-tolerated and cost-effective in emergency DCC.

Keywords: Atrial fibrillation, Cardioversion, Emergency procedures, Midazolam, Propofol, Sedation

\footnotetext{
* Correspondence: f.guerra@univpm.it

${ }^{1}$ Cardiology and Arrhythmology Clinic, Marche Polytechnic University,

University Hospital "Ospedali Riuniti Umberto I - Lancisi - Salesi", Via Conca

71, Ancona, Italy

Full list of author information is available at the end of the article
}

(C) The Author(s). 2020 Open Access This article is licensed under a Creative Commons Attribution 4.0 International License, which permits use, sharing, adaptation, distribution and reproduction in any medium or format, as long as you give appropriate credit to the original author(s) and the source, provide a link to the Creative Commons licence, and indicate if changes were made. The images or other third party material in this article are included in the article's Creative Commons licence, unless indicated otherwise in a credit line to the material. If material is not included in the article's Creative Commons licence and your intended use is not permitted by statutory regulation or exceeds the permitted use, you will need to obtain permission directly from the copyright holder. To view a copy of this licence, visit http://creativecommons.org/licenses/by/4.0/ The Creative Commons Public Domain Dedication waiver (http://creativecommons.org/publicdomain/zero/1.0/) applies to the data made available in this article, unless otherwise stated in a credit line to the data. 


\section{Background}

Direct current cardioversion (DCC) represents the most widely used and effective method to restore sinus rhythm in patients with persistent atrial fibrillation (AF) [1-3]. $\mathrm{DCC}$ is, however, a painful procedure that can cause pain comparable to a surgical incision [4]. It therefore requires both analgesia and deep sedation which, according to the American Society of Anaesthesiologists, consists in a drug induced reduction of the level of consciousness, during which patients can respond purposefully to painful stimuli [5]. The use of sedation during DCC can also play a role in reducing the pain-related catecholamine surge therefore preventing the recollection of such an unpleasant experience by the patient $[6,7]$.

To this day, no specific guidelines or recommendations with regards to the most appropriate drug that should be used for procedural sedation is available and different sedation strategies have been described in literature but no consensus on the most efficient strategy has been reached. One of the most commonly used agent for sedation in DCC, is propofol [8] an intravenous sedative hypnotic drug which can be administered only by personnel trained in advanced airways management [9]. In order to overcome such a limitation, non-anaesthesiologists, such as cardiologists, emergency physicians or nurses [10] have sought potential alternatives such as benzodiazepines. In particular, the use of a cardiologist-only approach to sedation with midazolam for elective DCC has been described in literature $[11,12]$ and demonstrated to be as tolerated and effective as propofol in elective procedures [13]. However, concerns have been raised on the use of a cardiologist-only, midazolam-based strategy in urgent or emergency DCC as haemodynamic instability and lack of time could precipitate the risk of stroke and serious adverse events $[9,14]$.

The aim of this prospective, open-blinded, randomized study is to compare the feasibility of sedation with midazolam, administered exclusively by a cardiologist, to an anaesthesiologist-assisted protocol with propofol in urgent or emergency DCC.

\section{Methods}

\section{Population and inclusion criteria}

The "Intravenous beNzodiazepine Safety and Tolerability in Emergency Atrial fibrillation Direct-current cardioversion" (INSTEAD) is a single centre, prospective, open blinded, randomized study included consecutive patients admitted to the Emergency Department or the University Hospital "Ospedali Riuniti Umberto I - Lancisi - Salesi” requiring urgent or emergency DCC. The present study follows a pilot trial [14] that has already been performed in order to assess clinical feasibility.

Emergency DCC was defined as DCC performed in haemodynamically compromised patients with new- onset AF, according to European guidelines [1]. Urgent DCC was defined as DCC performed within $2 \mathrm{~h}$ from patient's admission due to persistent invalidating symptoms such as chest pain, pre-syncope, or severe palpitations. No anti-arrhythmic drugs were given prior to DCC for pharmacological cardioversion.

The inclusion criteria consisted in age $>18$ years old and admission for high rate AF or atrial flutter requiring urgent/emergency cardioversion. A documented or suspected allergy or intolerance to midazolam or propofol represented the only exclusion criterion. No patient was excluded based on comorbidities or concomitant diseases in order to reflect "real-life" population data.

The study conforms to the Declaration of Helsinki and was performed in accordance with the Consolidated Standards of Reporting Trials (CONSORT) standards and national recommendations by competent authorities (Supplementary Table 1). The protocol was approved by the internal review board of our institution (University Hospital "Ospedali Riuniti Umberto I - Lancisi - Salesi”). Written informed consent was gained from all patients.

\section{Peri-procedural assessment}

An accurate medical history was recorded from all patients in order to assess cardiovascular risk factors such as hypertension, diabetes, dyslipidaemia, family history and smoking habit. In addition, we recorded the patients' comorbidities and known cardiovascular diseases. We also took note of the drug history and the number of previous cardioversions, electrical or pharmacological, that the patients had undergone. We performed routine blood tests, a 12 lead ECG and an echocardiogram for each patient.

\section{Cardioversion}

All enrolled patients were randomized in a 1:1 fashion into the propofol or midazolam group. The CONSORT flow diagram is showed in Fig. 1.

With regards to the propofol group, the procedure was carried out with the assistance of the anaesthesiologist who administered propofol $1 \mathrm{mg} / \mathrm{kg}$ followed by $0.5 \mathrm{mg} /$ kg every 3 min until satisfactory sedation (Ramsay sedation scale > 4) [15]. The cardiologist delivered the shock through a manual external defibrillator (Zoll M-Series, Zoll Medical Corporation, Chelmsford, MA, USA).

In the midazolam group, both procedural sedation and DCC were carried out by the cardiologist who administered a starting dose of midazolam $3 \mathrm{mg}$, followed by 2 mg every 2 min until satisfactory response (Ramsay sedation scale >4) [15]. The anaesthesiologist was readily available during the whole procedure in order to intervene if deemed necessary.

A maximum of three shocks were delivered, following a step up published protocol [16]: a first $150 \mathrm{~J}$ shock with 


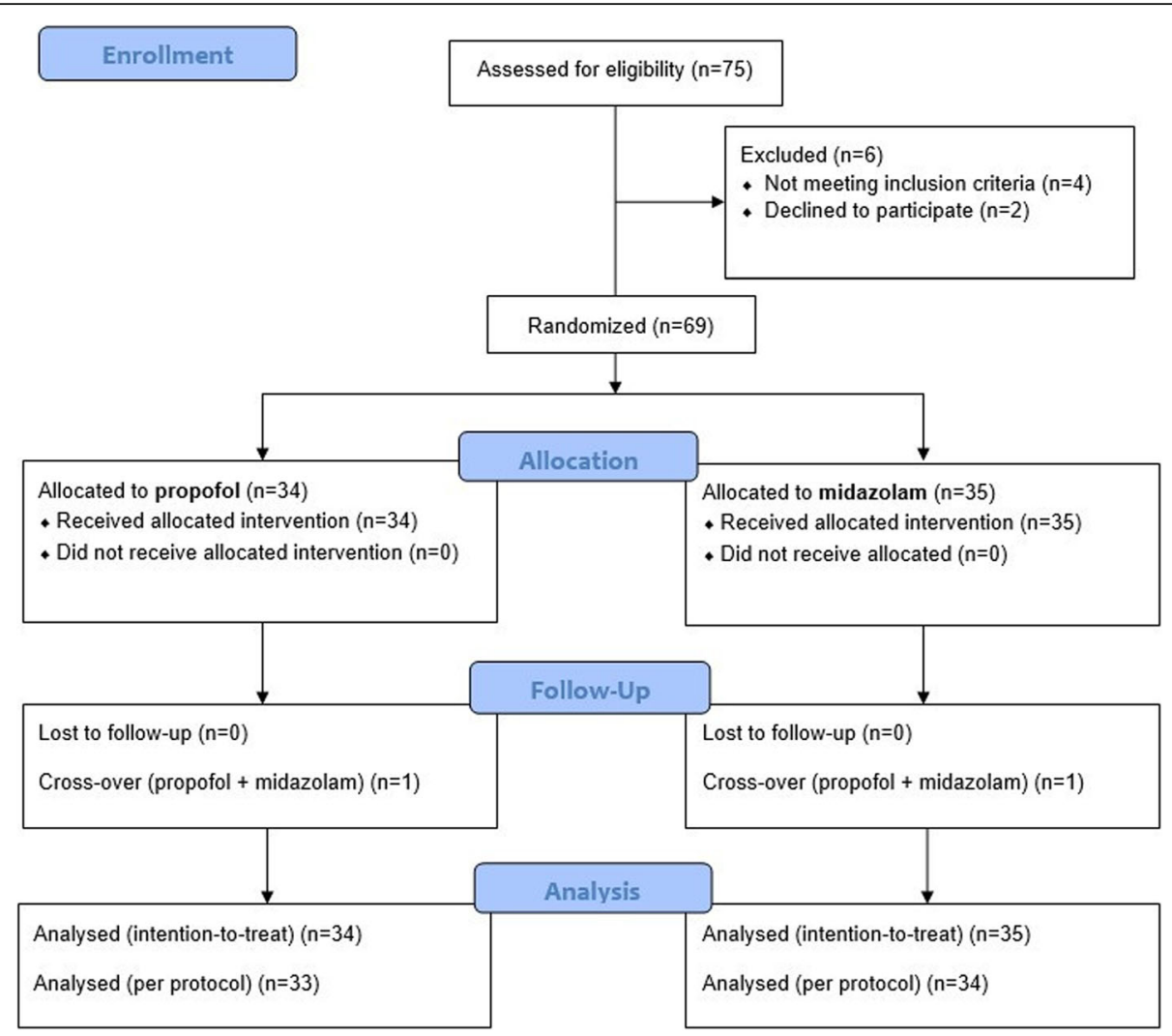

Fig. 1 The CONSORT flow diagram detailing enrolment procedure

manual paddles in antero-apical position. If not effective, a second shock of $200 \mathrm{~J}$ was delivered keeping the paddles in the same position. If both shocks failed to restore sinus rhythm, a third $200 \mathrm{~J}$ shock was delivered with adhesive patches in the anteroposterior position.

One-hundred percent oxygen supplementation and saline infusion were provided to each patient during the procedure.

At the end of the procedure, $1 \mathrm{mg}$ of flumazenil was administered to the patients in the midazolam group in order to revert the effects of the benzodiazepine.

When awake, all patients were asked to rate the pain and distress on a scale going from no pain to the worst possible pain and distress according to a visual analogue scale (VAS).

A 12-lead ECG was performed at the beginning and at the end of the procedure in order to assess the restoration of sinus rhythm and all vital status parameters (blood pressure, heart rate, saturation) as well as delay, length of procedure, length of monitoring and hospitalization were registered.

\section{Endpoints}

The primary safety endpoint was a composite of death and the following peri-procedural adverse events requiring medical intervention: bradycardia (defined as heart rate $<50 \mathrm{bpm}$ for at least $30 \mathrm{~s}$ ), severe hypotension (defined as systolic blood pressure $<80 \mathrm{mmHg}$ ), severe hypoxia (defined as oxygen saturation $<85 \%$ ), stroke, transient ischaemic attack and need for orotracheal intubation.

Other safety endpoints included the variation of blood pressure, heart rate and oxygen saturation registered before induction, before delivery of the shock, after the shock and after recovery. Tolerability of the procedure was assessed using the VAS.

Efficacy was assessed based on the number of very early recurrences (within $2 \mathrm{~h}$ ) and early recurrences (within 24 h). All patients discharged before $24 \mathrm{~h}$ from the Emergency Department were called by phone the day after the procedure in order to confirm stable sinus rhythm. Timerelated issues (delay and length of the procedure, monitoring time and length of hospitalization) were also considered.

Finally, we analysed the cost-effectiveness of the procedure carried out by the cardiologist alone with midazolam, compared to procedural sedation with propofol and anaesthesiologist assistance. Direct total costs were defined as the total of personnel costs, material costs and hospitalization costs. For a more detailed definition of costs calculation please refer to our previous study [13]. Indirect costs were not taken into account. 


\section{Statistical analysis}

Quantitative variables were checked for normality by the Kolmogorov-Smirnov test, and described as mean and standard deviation (if normally distributed) or median and first to third quartile (if not normally distributed). ANOVA adjusted by age and sex was used to compare normally distributed quantitative variables. KruskalWallis ANOVA adjusted by age and sex was used to compare non-normally distributed quantitative variables. Categorical variables were described by absolute number and assessed by using $\chi^{2}$ analysis. General linear model for repeated measures was used to assess timedependent changes of blood pressure, heart rate and oxygen saturation. As one patient per group ended up having both interventional drugs administered (Fig. 1), an intention-to-treat analysis was adopted.

Being a feasibility study, a formal, a-priori, sample size calculation was not performed. However, considering an expected composite endpoint rate of $18 \%$ from our pilot study [14], a population of $\geq 34$ subjects in each group would have a $>80 \%$ power to show that the primary safety endpoint (composite of death and the following peri-procedural adverse events) for the midazolam group was non-inferior to the control group, considering a preserved fraction (i.e. the fraction of the expected main effect that was estimated as clinically relevant) of 50\% [17] $(\alpha=0.05$, one-tail test). Therefore, an increase of more than $50 \%$ of the composite endpoint rate in the midazolam group when compared to the propofol group would have been deemed as inferior.

SPSS 25.0 for Windows (SPSS Inc., Chicago, IL, USA) was used for statistical analysis. Values of $p<0.05$ were taken as statistically significant.

\section{Results}

\section{General characteristics}

Sixty-nine patients were consecutively enrolled in our study, 61 of which $(84.4 \%)$ had atrial fibrillation and eight (11.6\%) were admitted with atrial flutter. General characteristics of the population, including risk factors, comorbidities, underlying heart disease, echocardiographic parameters and previous arrhythmic episodes are described in Table 1.

\section{Primary composite safety endpoint}

Eighteen patients (26.1\%) met the composite safety endpoint (Table 2). No deaths, neurological sequelae or need for orotracheal intubation were reported. All adverse events in the midazolam group were all managed successfully by the cardiologist with no need for anaesthesiology support or advanced airways management. Procedural sedation was optimally tolerated with propofol as well as with midazolam as all reported a VAS value of zero after DCC.

\section{Secondary safety endpoints}

With regards to the variation of vital status parameters patients in the propofol group experienced a greater, although not statistically significant, decrease in systolic $(p=.336)$ and diastolic $(p=.347)$ blood pressure than those treated with midazolam (Fig. 2a-b). The trends for both heart rate and oxygen saturation were similar in both group ( $p=.885$ and $p=.392$ respectively, Fig. 2c-d). As the figure shows, heart rate plummeted after the shock, indicating sinus rhythm being restored at a lower rate. In the midazolam group, no patient experienced resedation after flumazenil administration.

\section{Efficacy endpoint}

Ten recurrences were registered in the first $24 \mathrm{~h}$. Of those, six were registered in the propofol group (four very early, two early) and four were registered in the midazolam group (two very early and two early), with no significant difference between groups $(p=.692)$.

\section{Time-related endpoints}

DCC randomized to propofol took on average $4 \mathrm{~min}$ more to perform ( $16 \pm 7 \mathrm{~min}$ vs. $12 \pm 5 \mathrm{~min} ; p=.013)$ and were burdened by a 30 min higher delay which was needed to get more personnel ready $(43 \pm 11 \mathrm{~min}$ vs. $13 \pm 4 \mathrm{~min} ; p=.016$; Fig. 3a). Monitoring time did not differ between the two groups $(3.7 \pm 2.0 \mathrm{~h}$ for propofol vs. $2.9 \pm 1.4 \mathrm{~h}$ for midazolam; $p=.107$ ), nor did median hospitalization length (1.2 days for propofol vs. 1.1 days for midazolam; Fig. 3b).

\section{Costs analysis}

The median medical cost was of $16.4 €$ for the midazolam group and $47.8 €$ for the propofol group $(p<.001)$ and was mainly driven by an increased delay and lack of coordination between the cardiologist and the anaesthesiologist. In terms of material costs, the median cost in the midazolam group was higher than the propofol one as the former implied the use of flumazenil (83.7 $€$ vs. $78.8 €, p<.001)$. Hospitalization costs in the midazolam group added up to a median of $28.1 €$ and 48.7 for the propofol group $(p=.022)$. The total median cost of urgent/emergency DCC with midazolam was estimated to be $129.0 €$ (1st-3rd quartiles 114.6-151.6) and $195.6 €$ (1st-3rd quartiles 147.3-726.7) with propofol $(p<.001)$.

\section{Discussion}

Procedural sedation plays a key role in many procedures carried out for arrhythmia related issues such as device implant, electrophysiological studies, catheter ablations [18] and DCC [6]. As these procedures can be painful, an adequate level of sedation not only is required but can also reduce the pain-related catecholamine surge therefore preventing the recollection of an unpleasant 
Table 1 General characteristics

\begin{tabular}{|c|c|c|c|c|}
\hline Variable & Total population $(n=69)$ & Propofol group $(n=34)$ & Midazolam group $(n=35)$ & $P$-value \\
\hline Male gender (n, \%) & $45(65.2)$ & $20(58.8)$ & $25(71.4)$ & .272 \\
\hline Age (years) & $66.5 \pm 12.0$ & $67.9 \pm 12.6$ & $65.1 \pm 11.4$ & .322 \\
\hline BMI (kg/m2) & $28.5 \pm 5.2$ & $28.0 \pm 3.6$ & $29.1 \pm 6.1$ & .713 \\
\hline Hypertension (n, \%) & $49(71.0)$ & $26(76.5)$ & $23(65.7)$ & .325 \\
\hline Diabetes (n, \%) & $7(10.1)$ & $4(11.8)$ & $3(8.6)$ & .660 \\
\hline Dyslipidaemia (n, \%) & $28(40.6)$ & $13(38.2)$ & $15(42.9)$ & .696 \\
\hline Smoking habit (n, \%) & $16(23.2)$ & $7(20.6)$ & $9(25.7)$ & .614 \\
\hline $\operatorname{COPD}(n, \%)$ & $14(20.3)$ & $7(20.6)$ & $7(20.0)$ & .321 \\
\hline CRI $(n, \%)$ & $4(5.8)$ & $3(8.8)$ & $1(2.9)$ & .289 \\
\hline Thyroid disorders (n, \%) & $13(18.8)$ & $6(17.6)$ & $7(20.0)$ & .319 \\
\hline CHA2DS2-VASc score (median, 1st-3rd) & $2(1-3)$ & $3(2-3)$ & $2(1-3)$ & .649 \\
\hline Haemoglobin (g/dl) & $13.6 \pm 3.3$ & $13.5 \pm 3.1$ & $13.7 \pm 3.7$ & .806 \\
\hline eGFR (ml/min) & $85.7 \pm 33.6$ & $83.4 \pm 37.3$ & $88.0 \pm 29.7$ & .581 \\
\hline Sodium (mEq/l) & $134.2 \pm 29.1$ & $135.3 \pm 24.4$ & $133.0 \pm 33.4$ & .749 \\
\hline Potassium (mEq/l) & $4.0 \pm 1.0$ & $4.0 \pm 0.9$ & $3.9 \pm 1.0$ & 696 \\
\hline BNP $(p g / m l)$ & $257.2 \pm 86.4$ & $245.4 \pm 71.5$ & $269.2 \pm 99.9$ & .276 \\
\hline \multicolumn{5}{|l|}{ Type of heart disease: } \\
\hline Hypertensive & $40(58.0)$ & $19(55.9)$ & $21(60.0)$ & .729 \\
\hline Ischemic & $11(15.9)$ & $7(20.6)$ & $4(11.4)$ & .298 \\
\hline Valvular & $5(7.2)$ & $3(8.8)$ & $2(5.7)$ & .618 \\
\hline Idiopathic dilatative & $2(2.9)$ & $1(2.9)$ & $1(2.8)$ & .486 \\
\hline Lone AF & $11(15.9)$ & $4(11.8)$ & $7(20.0)$ & .350 \\
\hline \multicolumn{5}{|l|}{ Echographic characteristics: } \\
\hline $\mathrm{LAD}(\mathrm{mm})$ & $29.6 \pm 18.1$ & $30.4 \pm 19.0$ & $29.0 \pm 17.3$ & .749 \\
\hline LVEDD (mm) & $49.2 \pm 8.7$ & $49.3 \pm 7.7$ & $49.2 \pm 9.8$ & .965 \\
\hline LVESD (mm) & $30.1 \pm 6.4$ & $30.4 \pm 6.4$ & $31.6 \pm 6.4$ & .487 \\
\hline LVEF (\%) & $44.4 \pm 15.0$ & $44.7 \pm 14.9$ & $44.2 \pm 15.4$ & .930 \\
\hline \multicolumn{5}{|l|}{ AAD at enrolment: } \\
\hline Flecainide (n, \%) & $15(22.7)$ & $7(21.9)$ & $8(23.5)$ & .873 \\
\hline Propafenone (n, \%) & $4(6.1)$ & $2(6.2)$ & $2(5.8)$ & .933 \\
\hline Amiodarone (n, \%) & $5(7.6)$ & $3(9.4)$ & $2(5.9)$ & .592 \\
\hline$\beta$-blockers (n, \%) & $31(47.0)$ & $15(46.9)$ & $16(47.1)$ & .988 \\
\hline CCBs $(n, \%)$ & $18(27.3)$ & $10(31.3)$ & $8(23.5)$ & .482 \\
\hline Anti-platelet (n, \%) & $15(21.7)$ & $7(20.6)$ & $8(25.0)$ & .669 \\
\hline Anticoagulant (n, \%) & $52(75.4)$ & $26(76.5)$ & $26(74.3)$ & .869 \\
\hline Previous AF episodes (median, 1st-3rd) & $2(1-3)$ & $2(0-3)$ & $2(1-3)$ & .353 \\
\hline Previous DCC (median, 1st-3rd) & $0(0-1)$ & $0(0-2)$ & $0(0-1)$ & .267 \\
\hline Previous PC (median, 1st-3rd) & $0(0-1)$ & $0(0-1)$ & $0(0-1)$ & .844 \\
\hline
\end{tabular}

$A A D$ Anti-arrhythmic drug, $A F$ Atrial fibrillation, $B M I$ Body mass index, $B N P$ Brain natriuretic peptide, $C C B$ Calcium channel blocker, $C O P D$ Chronic obstructive pulmonary disease, CRI Chronic renal impairment, DCC Direct-current cardioversion, eGFR Estimated glomerular filtration rate, $L A D$ Left atrial diameter, LVEDD Left ventricular end-diastolic diameter, LVESD Left ventricular end-systolic diameter, LVEF Left ventricular ejection fraction, PC Pharmacological cardioversion

experience [13]. Sedation has been described as a continuum including progressive stages: minimal sedation or anxiolysis, moderate sedation/analgesia, deep sedation/analgesia and general anaesthesia [5].
The results of the present study confirm that procedural sedation with midazolam could be a viable alternative to the propofol-based protocol, even in an emergency/urgency setting. Both drugs showed a similar 
Table 2 primary safety endpoint

\begin{tabular}{|c|c|c|c|}
\hline Adverse events & $\begin{array}{l}\text { Propofol group } \\
(n=34)\end{array}$ & $\begin{array}{l}\text { Midazolam group } \\
(n=35)\end{array}$ & $\begin{array}{l}P \text { - } \\
\text { value }\end{array}$ \\
\hline Total (n, \%) & $10(29.4)$ & $8(22.9)$ & .535 \\
\hline Bradycardia (n, \%) & $3(8.8)$ & $5(14.3)$ & .479 \\
\hline $\begin{array}{l}\text { Severe hypotension ( } \mathrm{n} \text {, } \\
\%)\end{array}$ & $5(14.7)$ & $3(8.6)$ & .426 \\
\hline Severe hypoxia $(n, \%)$ & $2(5.9)$ & $0(0)$ & .145 \\
\hline $\begin{array}{l}\text { Neurologic event ( } \mathrm{n} \text {, } \\
\%)\end{array}$ & $0(0)$ & $0(0)$ & - \\
\hline $\begin{array}{l}\text { Orotracheal intubation } \\
(n, \%)\end{array}$ & $0(0)$ & $0(0)$ & - \\
\hline Death $(n, \%)$ & $0(0)$ & $0(0)$ & - \\
\hline
\end{tabular}

safety profile as no patient experienced death, neurological events or needed orotracheal intubation. The number of adverse events in the midazolam group were fewer than those experienced in the propofol group and more importantly all of them were easily managed by the cardiologist alone with no need of intervention or assistance by the anaesthesiologist. On a note, the number of adverse events and the recurrence rates during emergency/urgent DCC were higher when compared to events in elective procedures [13]. This result may be secondary to the fact that in an emergency/urgent setting a higher variation of vital status parameters may be seen, leading to patients that are more labile and prone to adverse events.

Regarding recurrences, the difference was not statistically significant between the two groups. However, it is important to underline that the rate of recurrence could be different in patients who undergo urgent or emergency DCC compared to elective procedures. In fact, arrhythmia recurred in $14 \%$ of our patients, more than what usually expected in elective DCC [19]. Potential hypotheses for the higher rate of recurrence could include high variation of vital parameters, higher rate of comorbidities and absence of pre-treatment with antiarrhythmic drugs in most of our patients.

The procedure carried out with the benzodiazepine was well tolerated as demonstrated by the fact that all patients scored a zero on the VAS scale. We feel that such a result is not only secondary to the sedation obtained with midazolam, but also partly related to the anterograde amnesia caused by the drug. Moreover, as described above, while oxygen saturation and heart rate had a similar trend in both study groups, patients who received propofol experienced a more important drop in systolic blood pressure than in those in the midazolam group. This data confirm the effect of propofol as a peripheral vasodilator, therefore causing a greater impact on haemodynamic parameters [20].

In addition to this, the results clearly show how the length of the procedure and the associated delays can be shortened by using midazolam instead of propofol. We believe that the use of flumazenil as an antagonist to midazolam is the key factor contributing to a reduced procedural time. Flumazenil has been described to be a safe and effective reversal agent [21] and allows a shorter recovery period. However, given the difference in the half-life between midazolam and its antagonist, concerns have been expressed regarding potential re-sedation [22]. Our data support the safety of flumazenil, with no patients experiencing re-sedation during monitoring time or after discharge.

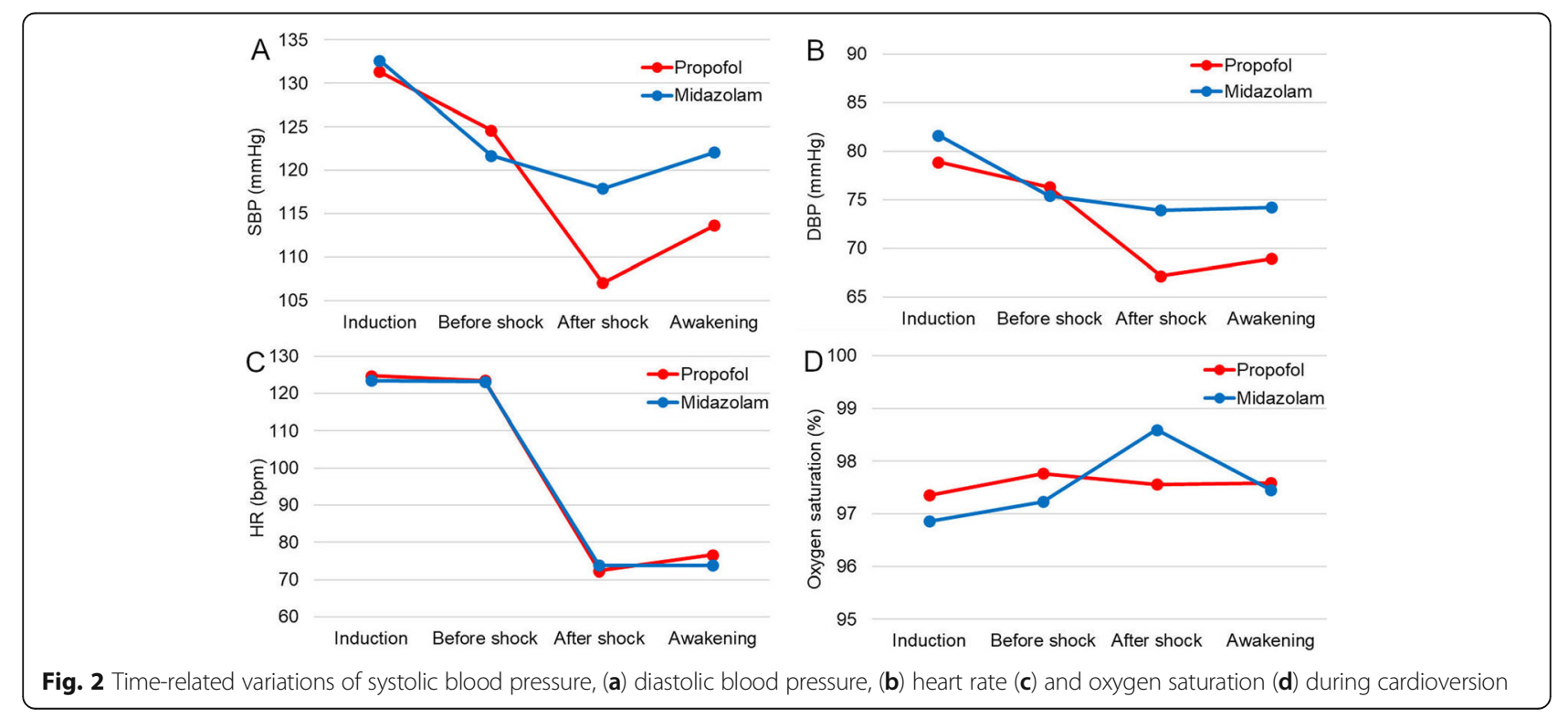




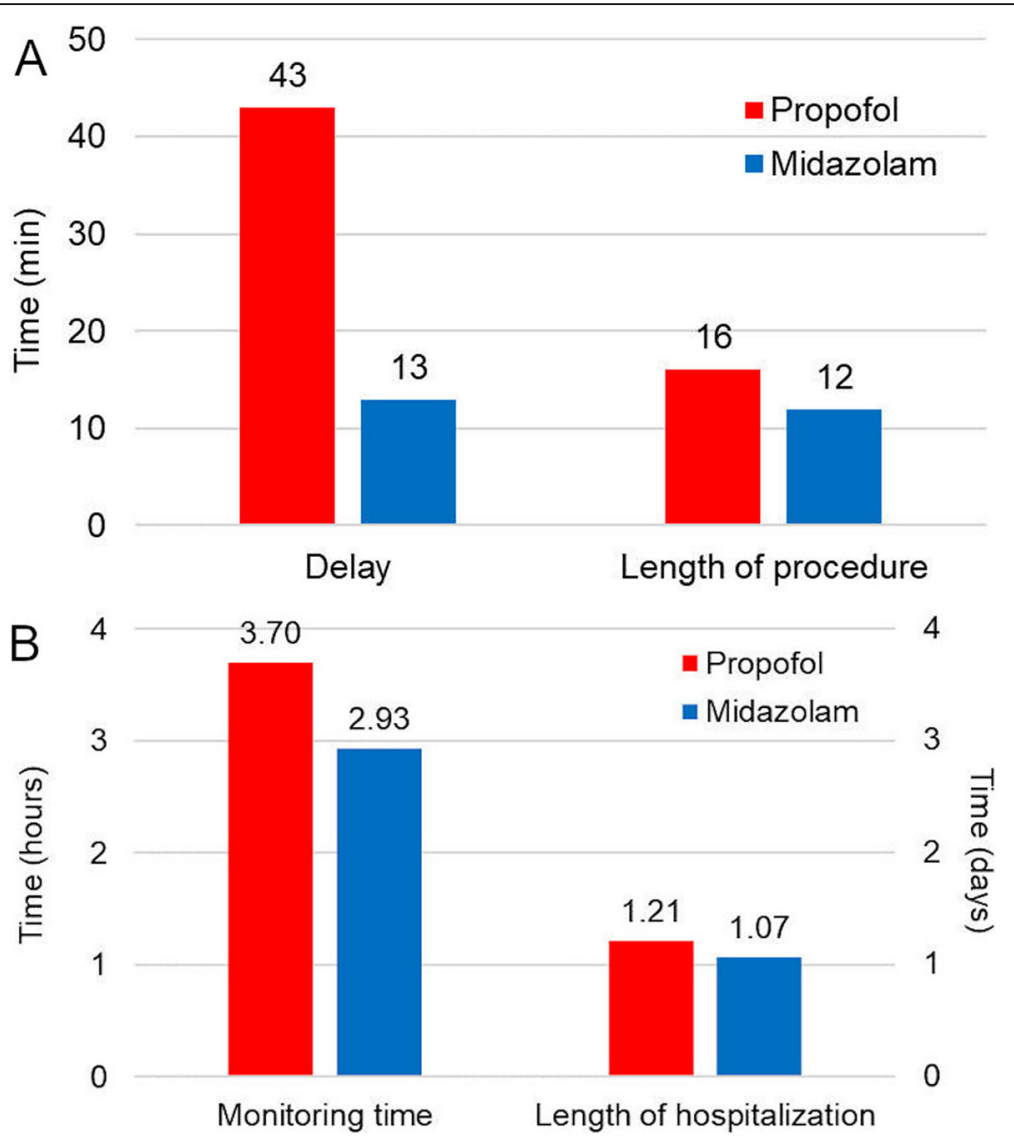

Fig. 3 Differences in procedural times: (a) procedural delay and procedural length; (b) monitoring time and total in-hospital time

Our findings show that the use of midazolam can reduce costs by decreasing the time spent for the procedure itself and by having fewer operators needed to perform the procedure, as the cardiologist can safely manage both sedation and shock delivery. Standardizing a protocol which does not imply the need for anaesthesiologist support appears cost-effective.

\section{Limitations}

Some limitations of the present trial deserve to be discussed. Firstly, double-blinding was deemed technically unfeasible due to the different number of physicians involved, the different intravenous formulations and the different titration protocols. Second, differences in experience and expertise of the cardiologists with midazolam could have provided an unintentional bias, albeit very difficult to measure. Third, the lower-than-expected difference in the composite endpoint between the two groups leaves open the chance of a type II error, even if of minor magnitude from a clinical point of view. However, it must be stressed out that the rate of severe complications was too low to allow for any firm conclusion regarding safety, being this a feasibility study with a relatively small sample size. Fourth, the present results could be difficult to extrapolate in other clinical settings, especially in those countries in which cardiologists are trained in advanced airways management or in which DCC are not primarily managed by the cardiologist [10].

Finally, we found that one patient who regularly took benzodiazepines for insomnia had built a tolerance which limited our possibility of using midazolam and had to be crossed over to propofol. Nonetheless, we also had one patient who probably metabolized propofol too quickly and as a consequence midazolam had to be used for sedation. However, we feel that this examples strongly confirm the need of finding easily manageable, safe and well tolerated options for procedural sedation.

\section{Conclusions}

A cardiologist-only approach to procedural sedation with midazolam could represent a feasible and well tolerated option for DCC even in the emergency/urgency setting. Adverse events were managed by the cardiologist in both groups with no need for intervention by the anaesthesiology team. The length of procedure was shortened when midazolam was used and fewer delays were registered, thus potentially leading to reduced costs. 


\section{Supplementary information}

Supplementary information accompanies this paper at https://doi.org/10. 1186/s12872-020-01664-1.

Additional file 1: Supplementary Table 1. CONSORT 2010 checklist of information to include when reporting a randomised trial.

\section{Abbreviations}

AF: Atrial fibrillation; DCC: Direct current cardioversion;

ECG: Electrocardiogram; VAS: Visual analogical scale

\section{Acknowledgements}

None.

\section{Authors' contributions}

GS and FG made substantial contributions to the conception and design of the work; the acquisition, analysis, and interpretation of data; the paper draft and its revision. AC, ADR, EA, AC, MC and AD made substantial contributions to the acquisition of data and the revision of the paper. All authors have approved the submitted version and have agreed both to be personally accountable for the author's own contributions and to ensure that questions related to the accuracy or integrity of any part of the work.

\section{Funding}

This research did not receive any specific grant from funding agencies in the profit or not-for-profit sectors.

\section{Availability of data and materials}

The dataset supporting the conclusions of this article will be available upon request to the corresponding author.

\section{Ethics approval and consent to participate}

The study conforms to the Declaration of Helsinki and was performed in accordance with the Consolidated Standards of Reporting Trials (CONSORT) standards and national recommendations by competent authorities. The protocol was approved by the internal review board of our Hospital. Written informed consent was gained from all patients.

\section{Consent for publication}

Not applicable.

\section{Competing interests}

Prof. Federico Guerra is an Associate Editor of BMC Cardiovascular Diseases. All other authors declare they have no conflicts of interest related to the present manuscript.

\section{Author details}

${ }^{1}$ Cardiology and Arrhythmology Clinic, Marche Polytechnic University, University Hospital "Ospedali Riuniti Umberto I - Lancisi - Salesi", Via Conca 71, Ancona, Italy. ${ }^{2}$ Anaesthesia and Intensive Care Unit, Marche Polytechnic University, University Hospital "Ospedali Riuniti", Ancona, Italy.

Received: 20 May 2020 Accepted: 10 August 2020

Published online: 25 August 2020

\section{References}

1. Kirchhof P, Benussi S, Kotecha D, Ahlsson A, Atar D, Casadei B, et al. 2016 ESC Guidelines for the management of atrial fibrillation developed in collaboration with EACTS: The Task Force for the management of atria fibrillation of the European Society of Cardiology (ESC) Developed with the special contribution of the Europea. Eur Heart J. 2016;37(September):2893962. https://doi.org/10.1093/eurheartj/ehw210.

2. Hellman T, Kiviniemi T, Vasankari T, Nuotio I, Biancari F, Bah A, et al. Prediction of ineffective elective cardioversion of atrial fibrillation: a retrospective multi-center patient cohort study. BMC Cardiovasc Disord. 2017;17:33. https://doi.org/10.1186/s12872-017-0470-0.

3. Jarman JWE, Hussain W, Wong T, Markides V, March J, Goldstein L, et al. Resource use and clinical outcomes in patients with atrial fibrillation with ablation versus antiarrhythmic drug treatment. BMC Cardiovasc Disord. 2018;18:211. https://doi.org/10.1186/s12872-018-0946-6.
4. Mitchell-Hines T, Ellison K, Willis S. Using bispectral index monitoring to gauge depth of sedation/analgesia. Nurs Crit Care. 2017;12:12-6. https://doi. org/10.1097/01.CCN.0000511003.39965.d2.

5. Gross JB, Bailey PL, Connis RT, Coté CJ, DavisFG, Epstein BS, Gilbertson L, Nickinovich DG, Zerwas JM, Zuccaro G, Jr. Practice guidelines for sedation and analgesia by non-anesthesiologists. A report by the American Society of Anesthesiologists Task Force on Sedation and Analgesia by NonAnesthesiologists. Anesthesiology. 1996;84:459-71.

6. Guerra F, Stronati G, Capucci A. Sedation in cardiac arrhythmias management. Expert Rev Cardiovasc Ther. 2018;16:163-73. https://doi.org/ 10.1080/14779072.2018.1429914.

7. Swann A, Williams J, Fatovich DM. Recall after procedural sedation in the emergency department. Emerg Med J. 2007;24:322-4.

8. Reed SS, Lewis SR, Nicholson A, Alderson P, Smith AF. Anaesthetic and sedative agents used for electrical cardioversion. Cochrane Database Syst Rev. 2015;2015(3):CD010824. https://doi.org/10.1002/14651858.CD010824. pub2.

9. Perel A. Non-anaesthesiologists should not be allowed to administer propofol for procedural sedation: a consensus statement of 21 European National Societies of Anaesthesia. Eur J Anaesthesiol. 2011:28:580-4

10. Boodhoo L, Bordoli G, Mitchell AR, Lloyd G, Sulke N, Patel N. The safety and effectiveness of a nurse led cardioversion service under sedation. Heart. 2004;90:1443-6.

11. Notarstefano P, Pratola C, Toselli T, Baldo E, Ferrari R. Sedation with midazolam for electrical cardioversion. Pacing Clin Electrophysiol. 2007;30: 608-11.

12. Morani G, Bergamini C, Angheben C, Pozzani L, Cicoira M, Tomasi L, et al. General anaesthesia for external electrical cardioversion of atrial fibrillation: experience of an exclusively cardiological procedural management. Europace. 2010;12:1558-63. https://doi.org/10.1093/europace/euq276.

13. Guerra F, Pavoni I, Romandini A, Baldetti L, Matassini MV, Brambatti M, et al. Feasibility of a cardiologist-only approach to sedation for electrical cardioversion of atrial fibrillation: a randomized, open-blinded, prospective study. Int J Cardiol. 2014;176:930-5. https://doi.org/10.1016/j.ijcard.2014.08.050.

14. Guerra F, Stronati G, Mariotti G, Capucci A. Safety and tolerability of a cardiologist-only approach to sedation for electrical cardioversion in the Emergency Department: the INSTEAD pilot trial. Int J Cardiol. 2016;223:7667. https://doi.org/10.1016/j.jicard.2016.08.291.

15. Ramsay MA, Savege TM, Simpson BR, Goodwin R. Controlled sedation with alphaxalone-alphadolone. Br Med J. 1974;2:656-9. https://doi.org/10.1136/ bmj.2.5920.656.

16. Kirchhof P, Eckardt L, Loh P, Weber K, Fischer RJ, Seidl KH, et al. Anteriorposterior versus anterior-lateral electrode positions for external cardioversion of atrial fibrillation: a randomised trial. Lancet. 2002:360:1275-9.

17. Althunian TA, de Boer $\mathrm{A}$, Groenwold $\mathrm{RHH}$, Klungel $\mathrm{OH}$. Defining the noninferiority margin and analysing noninferiority: an overview. $\mathrm{Br} J \mathrm{Clin}$ Pharmacol. 2017;83:1636-42. https://doi.org/10.1111/bcp.13280.

18. Ding J, Xu J, Ma W, Chen B, Yang P, Qi Y, et al. A novel individualized strategy for cryoballoon catheter ablation in patients with paroxysmal atrial fibrillation. BMC Cardiovasc Disord. 2019;19:299. https://doi.org/10.1186/ s12872-019-01295-1.

19. Boriani G, Diemberger I, Biffi M, Domenichini G, Martignani C, Valzania C, et al. Electrical cardioversion for persistent atrial fibrillation or atrial flutter in clinical practice: predictors of long-term outcome. Int J Clin Pract. 2007;61: 748-56.

20. Kaye P, Govier M. Procedural sedation with propofol for emergency DC cardioversion. Emerg Med J. 2014;31:904-8. https://doi.org/10.1136/ emermed-2013-202742.

21. Lee SP, Sung I-K, Kim JH, Lee S-Y, Park HS, Shim CS. Efficacy and safety of flumazenil injection for the reversal of midazolam sedation after elective outpatient endoscopy. J Dig Dis. 2018;19:93-101.

22. Coll-vinent B, Sala X, Bragulat E, Espinosa G. Sedation for cardioversion in the Emergency Department: analysis of effectiveness in four protocols. Ann Emerg Med. 2003;42(December):767-72.

\section{Publisher's Note}

Springer Nature remains neutral with regard to jurisdictional claims in published maps and institutional affiliations. 